\title{
Experimental conditions required for accurate measurements of electrical resistivity, thermal conductivity, and dimensionless figure of merit (ZT) using Harman and impedance spectroscopy methods
}

Cite as: J. Appl. Phys. 125, 025111 (2019); https://doi.org/10.1063/1.5077071

Submitted: 24 October 2018 . Accepted: 22 December 2018 . Published Online: 11 January 2019

Braulio Beltrán-Pitarch (D), Jesús Prado-Gonjal, Anthony V. Powell, and Jorge García-Cañadas (D)

\section{ARTICLES YOU MAY BE INTERESTED IN}

Thermal conductivity, electrical resistivity, and dimensionless figure of merit (ZT) determination of thermoelectric materials by impedance spectroscopy up to $250^{\circ} \mathrm{C}$ Journal of Applied Physics 124, 025105 (2018); https://doi.org/10.1063/1.5036937

Non-linear impedance spectroscopy for complete thermoelectric characterization: Beyond the zT estimation

Journal of Applied Physics 124, 235106 (2018); https://doi.org/10.1063/1.5063419

A practical field guide to thermoelectrics: Fundamentals, synthesis, and characterization Applied Physics Reviews 5, 021303 (2018); https://doi.org/10.1063/1.5021094

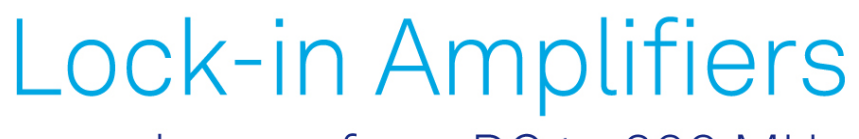
... and more, from DC to $600 \mathrm{MHz}$ Watch 


\title{
Experimental conditions required for accurate measurements of electrical resistivity, thermal conductivity, and dimensionless figure of merit ( $Z T)$ using Harman and impedance spectroscopy methods
}

\author{
Cite as: J. Appl. Phys. 125, 025111 (2019); doi: 10.1063/1.5077071 \\ Submitted: 24 October 2018 . Accepted: 22 December 2018. \\ Published Online: 11 January 2019
}

Braulio Beltrán-Pitarch, ${ }^{7}$ (D) Jesús Prado-Gonjal, ${ }^{2}$ Anthony V. Powell, ${ }^{2}$ and Jorge García-Cañadas ${ }^{1, a)}$ (D)

\begin{abstract}
AFFILIATIONS
${ }^{1}$ Department of Industrial Systems Engineering and Design, Universitat Jaume I, Campus del Riu Sec, 12071 Castellón, Spain

${ }^{2}$ Department of Chemistry, University of Reading, RG6 6AD Reading, United Kingdom
\end{abstract}

${ }^{\text {a)} E-m a i l: ~ g a r c i a j @ u j i . e s ~}$

\begin{abstract}
The Harman method is used extensively for the characterization of the dimensionless figure of merit ZT of thermoelectric (TE) materials and devices. However, its accuracy has often been questioned, since in many cases there are relatively high errors associated with the method. The impedance spectroscopy technique, which has recently been shown as a suitable tool to also characterize TE materials and devices, has some similarities with the Harman method and can also directly provide ZT. In order to obtain reliable measurements in both methods, there are some common critical points that must be taken into account, such as the requirement of fully adiabatic conditions and a negligible Joule effect. In this study, we have evaluated the effect of different experimental conditions in the accuracy of both methods using a sample with known TE properties. Our analysis has led to the identification of different sources of errors and other issues that have not been clearly identified to date that can lead to inaccurate results, namely, the need of a homogeneous Peltier effect at the junctions, problems arising from the use of Ag paint, and the selection of the right value for the current perturbation applied to the system. These problems and sources of errors need to be identified and carefully considered if accurate results are to be obtained.
\end{abstract}

Published under license by AIP Publishing. https:/ /doi.org/10.1063/1.5077071

\section{INTRODUCTION}

The Harman method $^{1}$ is a widely used technique for the determination of the dimensionless figure of merit ZT of thermoelectric (TE) materials ${ }^{2}$ and devices. ${ }^{3}$ The method typically requires two simple measurements, one under a high frequency current amplitude (ac condition) and the other once the steady state is achieved after applying a constant current (dc condition). These two measurements can be performed using a single equipment and sample setup, which is less time consuming and introduces lower uncertainties than the independent determination of all the TE properties that define ZT (the Seebeck coefficient, the electrical resistivity, and the thermal conductivity). However, the accuracy of the Harman method has frequently been questioned, as it requires certain experimental conditions that are often difficult to achieve, to be fulfilled. Several of these conditions have already been identified and have even led to modifications of this method. For example, the Joule effect can significantly influence the measurements. This led to the development of the advanced Harman method, ${ }^{4}$ where the influence of the Joule effect can be separated. In addition, adiabatic conditions are required, which are difficult to achieve due to heat losses by convection, 
radiation, or through the wires used to perform the measurements in the setup. ${ }^{5,6}$ Moreover, a uniform current flow through the sample is required for proper measurement of the ohmic resistance (ohmic voltage drop) of the sample. Together, these problems can lead to measurement errors of up to $35 \%$.

Impedance spectroscopy has recently attracted attention as a suitable alternative method for the characterization of TE materials and devices. ${ }^{8-13}$ This technique, in contrast to the Harman method, measures a wide range of frequencies and not only ac and de conditions. However, it has similarities with the Harman method, in the sense that ZT can be directly obtained from the high frequency and low frequency limits of the impedance spectrum. The impedance method also requires similar experimental conditions to determine ZT accurately and can be affected by the Joule effect, ${ }^{12}$ and convection and radiation losses as well. ${ }^{13,14}$

Here, we have evaluated the effect on the accuracy of both Harman and impedance methods of several experimental conditions, which have not been clearly identified previously. This investigation has been performed employing a skutterudite sample, whose TE properties were previously determined by established commercial instruments. The conditions that were evaluated comprise (i) the need of a homogeneous Peltier effect at the junctions, (ii) the influence of the employed current amplitude, (iii) the frequency used for the ac measurement in the Harman method, and (iv) the use of silver paint at the contacts. All of them are critical to reach an adequate characterization, although the frequency chosen for the ac measurement in the Harman method has a relatively minor influence. Consequently, minimizing these effects is key to obtain accurate results.

\section{SIMILARITIES BETWEEN THE HARMAN AND IMPEDANCE METHODS}

The Harman method consists of extracting the pure ohmic response and the Seebeck voltage contribution when a constant current is applied to a TE material or device. Figure 1(a) shows one of the two main ways to determine both contributions, in which the voltage difference variation is measured when a constant current, initially applied to the sample, is switched off [see the inset of Fig. 1(a)]. A voltage, $\mathrm{V}_{\mathrm{DC}}$, is achieved after reaching the steady state when the constant current is applied. When the current is switched off, an immediate drop in the voltage $\left(\mathrm{V}_{\mathrm{AC}}\right)$ is produced, caused by the ohmic resistance of the sample [see Fig. 1(a)]. After some seconds, the temperature difference, induced in the sample by the Peltier effect originating from the constant current, vanishes, the voltage tends to zero, and $V_{\mathrm{TE}}$ can be calculated, which is the voltage drop due to the Seebeck effect induced by the temperature difference. The other way to perform the Harman method differs only in how the ohmic contribution is measured, which in the other case is obtained by performing an ac current measurement at a high frequency, which ensures that a temperature difference is not present. From this ac measurement, the ohmic contribution $\left(\mathrm{V}_{\mathrm{AC}}\right)$ can be extracted directly. Frequency values from $15.9 \mathrm{~Hz}$ to $110 \mathrm{~Hz}$ are typically used. ${ }^{2,5}$ In both ways of application of the Harman method, the dimensionless figure of merit ZT of the TE material can be obtained by

$$
\mathrm{ZT}=\frac{\mathrm{V}_{\mathrm{TE}}}{\mathrm{V}_{\mathrm{AC}}} .
$$

Unlike the Harman method, impedance spectroscopy applies a sinusoidal current of certain amplitude to the system, which induces a voltage wave that is recorded. From the ratio of the amplitudes of the two waves (voltage/ current) and their phase shift, a point is generated in the complex plane (impedance spectrum). This experiment is repeated varying the frequency of the sinusoidal current, which may range from a few $\mathrm{mHz}$ to some $\mathrm{kHz}$. At each frequency, a point is generated, and all the points finally form the impedance spectrum [see Fig. 1(b)]. The high frequency intercept of the spectrum with the real axis $\left(Z^{\prime}\right)$ provides the ohmic resistance of the system $\left(R_{A C}\right)$, since at this point there is no phase shift and the heat is released and removed too quickly at both junctions for a temperature difference to be established. This parameter is basically similar to $V_{A C}$ in the Harman method. By contrast, when the frequency is very low, the temperature difference is established and a contribution to $R_{A C}$ appears due to the temperature difference induced Seebeck voltage $\left(R_{T E}\right)$, which can be calculated from the difference between the low and high frequency intercepts with the real axis of the spectrum [see Fig. 1(b)]. The determination of $\mathrm{V}_{\mathrm{TE}}$ in the Harman method is similar to the $R_{T E}$ obtained by impedance. At these very low frequencies, it is considered that the dc regime (steady state) is reached, and the total resistance of the system is given by $R_{D C}=R_{A C}+$ $R_{T E}$ [see Fig. 1(b)]. In a similar way to the Harman method, the dimensionless figure of merit $\mathrm{ZT}$ of the material can be determined as

$$
\mathrm{ZT}=\frac{\mathrm{R}_{\mathrm{TE}}}{\mathrm{R}_{\mathrm{AC}}} .
$$

\section{EXPERIMENTAL SETUP}

Figure 2 shows the most frequently adopted setups to perform the Harman method [Figs. 2(a) and 2(b)] and the setup used in our previous work ${ }^{12}$ to characterize TE materials by impedance spectroscopy [Fig. 2(c)]. The latter was shown to provide a suitable characterization when compared with commercial equipment results. ${ }^{12}$ Unless otherwise stated, all the results shown in this article were obtained employing the same setup [Fig. 2(c)].

It consists of a TE sample which is sandwiched between two copper pieces of the same cross-sectional area and of $2 \mathrm{~mm}$ thickness. This ensures a homogeneous current flow through the junctions and the TE sample. To provide a suitable electrical and thermal contact between the TE sample and the copper contacts, both materials were carefully polished with 1200 grit size silicon carbide sandpaper 

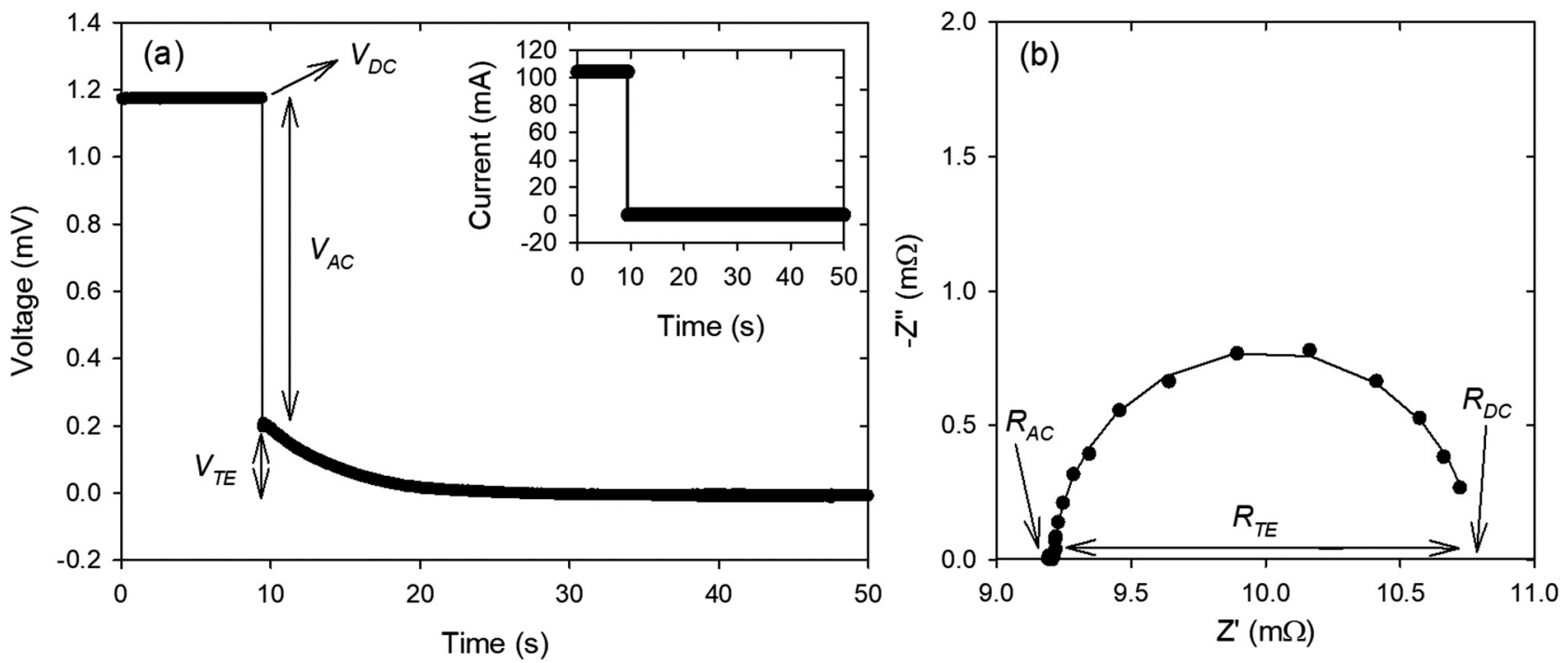

FIG. 1. (a) Voltage response obtained when the Harman method was performed on a $2.30 \mathrm{~mm} \times 2.11 \mathrm{~mm} \times 5.01 \mathrm{~mm}$ n-type skutterudite $\left(\mathrm{CoSb}_{2.75} \mathrm{Sn}_{0.05} \mathrm{Te}_{0.20}\right)$ at $50{ }^{\circ} \mathrm{C}$. The inset shows the $104 \mathrm{~mA}$ current step applied to the sample. (b) Impedance spectroscopy measurement from $5 \mathrm{mHz}$ to $10 \mathrm{kHz}$ performed on the same material and employing the same ac current amplitude (104 mA) as in the current step.

(Ref. 30-5208-012, Buehler), cleaned with acetone, and interconnected with $\mathrm{Ga}_{62} \mathrm{In}_{22} \mathrm{Sn}_{16}$ liquid metal (Ref. 14634, Alfa Aesar), which was added at both junctions. Two very thin copper wires of $15 \mu \mathrm{m}$ diameter were inserted in both junctions for the measurement of the voltage difference across the TE material. The small diameter of the wires is important in order to minimize heat losses by heat conduction through (a)

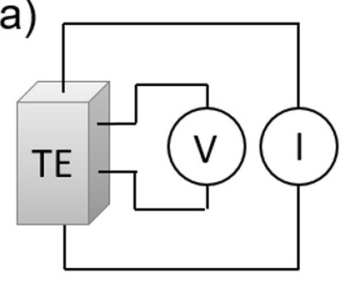

(b)

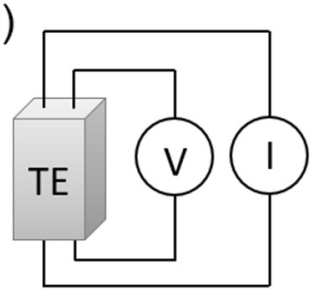

(c) Stainless

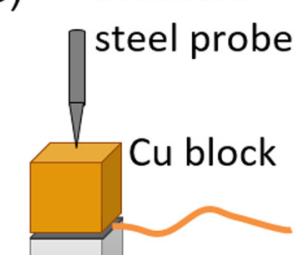

TE sample

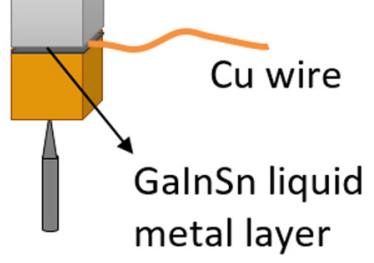

FIG. 2. (a) and (b) Schematic of typical 4-probe configurations to perform the Harman method or impedance spectroscopy measurements, and (c) detailed sketch of the optimized setup used in this study. them and to avoid producing separation between the two surfaces under contact. The current is applied to the copper blocks using two screws of stainless-steel, which is a material of relatively low thermal conductivity $[\approx 14 \mathrm{~W} /(\mathrm{m} \mathrm{K})]$. The screws were sharpened in order to minimize heat losses by conduction as well. A band heater (Ref. MB2E2JN1-B12, Watlow) was placed around the whole assembly to provide different ambient temperatures. The ambient temperature was measured with a K-type thermocouple placed close to the TE sample, which was controlled by a temperature controller (Watlow EZ Zone PM).

All the measurements were performed at $50^{\circ} \mathrm{C}$ ambient temperature inside a stainless steel vacuum chamber at a pressure lower than $10^{-4}$ mbar in order to suppress the influence of convection. Two TE samples were cut from a tetragonal and isotropic n-type skutterudite disc pellet $\left(\mathrm{CoSb}_{2.75} \mathrm{Sn}_{0.05} \mathrm{Te}_{0.20}\right)$ with dimensions of $2.30 \mathrm{~mm} \times 2.11 \mathrm{~mm} \times$ $5.01 \mathrm{~mm}$ (short sample, SS) and $2.13 \mathrm{~mm} \times 2.27 \mathrm{~mm} \times 6.90 \mathrm{~mm}$ (long sample, LS). They were cut with a diamond wire saw of $0.3 \mathrm{~mm}$ diameter to obtain a uniform and precise cut. Before the disc was cut, it was characterized using a Linseis LSR-3 apparatus to obtain its electrical conductivity and Seebeck coefficient, and by a Netzsch LFA 447 laser flash to determine the thermal diffusivity and specific heat. The sample thermal conductivity was calculated using the previous parameters (thermal diffusivity and specific heat) and the measurement of the mass density, which was performed using an Archimedes balance. To carry out the Harman method and impedance spectroscopy measurements, a PGSTAT30 potentiostat (Metrohm Autolab B. V.) equipped with a FRA2 impedance analyzer module and a BOOSTER10A 

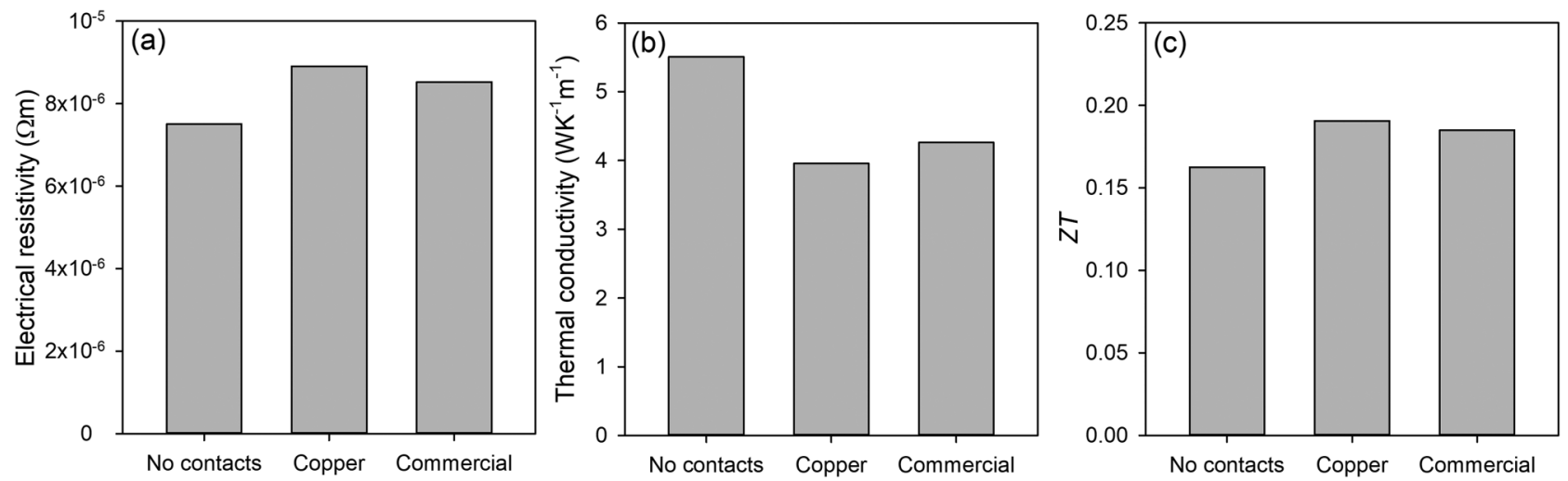

FIG. 3. (a) Electrical resistivity, (b) thermal conductivity, and (c) dimensionless figure of merit ZT measured by impedance spectroscopy without copper blocks (No contacts), with copper blocks (Copper), and measured using the commercial equipment (Commercial). Measurements were performed with the LS skutterudite sample.

was used. This equipment was controlled by the Nova 1.11 software. All the impedance measurements were performed at $0 \mathrm{~A}$ dc current and different ac current amplitudes, which will be indicated in Sec. IV.

\section{RESULTS AND DISCUSSION}

\section{A. Requirement of homogeneous Peltier effect and current}

To evaluate the importance of the existence of a homogeneous current and Peltier effect at the $\mathrm{Cu} / \mathrm{TE}$ junctions, the electrical resistivity [Fig. 3(a)], the thermal conductivity [Fig. 3(b)], and the dimensionless figure of merit ZT [Fig. 3(c)] were determined by impedance spectroscopy with [setup of Fig. 2(c)] and without [setup of Fig. 2(b)] the presence of $\mathrm{Cu}$ blocks for the LS skutterudite employing a $104 \mathrm{~mA}$ ac current. The electrical resistivity $\rho=\mathrm{R}_{\mathrm{AC}} \mathrm{A} / \mathrm{L}$ was determined from $R_{A C}$ and the area $A$ and length $L$ of the sample. The thermal conductivity $\lambda_{\mathrm{TE}}=\mathrm{S}^{2} \mathrm{TL} /\left(\mathrm{R}_{\mathrm{TE}} \mathrm{A}\right)$ was evaluated from $R_{T E}$, the ambient temperature $T$, the sample dimensions, and the Seebeck coefficient value $S$, measured by the commercial equipment. ${ }^{12} \mathrm{ZT}$ was obtained using Eq. (2). The results were compared with the data obtained using the commercial equipment.

It is observed from Fig. 3(a) that when no copper contacts were used to ensure a uniform current flow through the TE material, the electrical resistivity is underestimated by $12.0 \%$ with respect to the commercial equipment result. This underestimation can be attributed to the non-uniform current density existing at the positions where the voltage probes are located [see Fig. 2(b)], since the current is injected through the tips of point probes at the same plane where the voltage probes are. Measurements with copper contacts show a significantly lower deviation (4.1\%) versus the commercial equipment value. This may be due to small contributions from the electrical contact resistances at both junctions, since the $\mathrm{Cu}$ wires are inserted at the junctions and not contacted at the sides of the sample.

For the case of the thermal conductivity [Fig. 3(b)], a $29.2 \%$ deviation with respect to the commercial equipment value is found when no copper contacts are present. This is also due to a non uniform Peltier effect occurring at the junctions, since the electrical current is injected through the tip of the stainless steel probes, which only represents a small portion of the whole cross-sectional area of the sample and leads to an unsuitable lower value of $R_{T E}$. A more suitable result (3.0\% deviation) is found when copper contacts are included in the setup.

The deviations observed in the measurement of the electrical resistivity $\left(R_{A C}\right)$ and $R_{T E}$, resulting from the absence of $\mathrm{Cu}$ blocks in the setup, influence the accurate determination of ZT [see Eq. (2)], as reflected in Fig. 3(c). For the case without copper contacts, ZT is underestimated by $12.1 \%$ compared with the commercial equipment results and by a smaller amount $(6.7 \%)$ for the case with $\mathrm{Cu}$ contacts. The deviation in the ZT with no copper contacts is high but somewhat lower than expected, since the higher $R_{T E}$ value is partially compensated by the underestimation of the electrical resistivity (lower value of $R_{A C}$ ). These results clearly demonstrate the need of a uniform Peltier effect at the junctions in order to obtain proper measurements.

\section{B. Current pulse amplitude optimization}

Figure 4(a) shows the ohmic $\left(\mathrm{R}_{\mathrm{AC}}\right)$ and TE resistances for the Harman method and impedance spectroscopy measurements performed at different current amplitudes $(52,78,104$, $130,156,182,208,234$, and $260 \mathrm{~mA}$ ) for the SS skutterudite. For the Harman method measurements, the same values of the current amplitudes were used, but in this case, they correspond to the constant current applied which is later removed [see the inset of Fig. 1(a)]. Accordingly, the TE resistance refers in this case to the ratio between $V_{A C}$ and the 

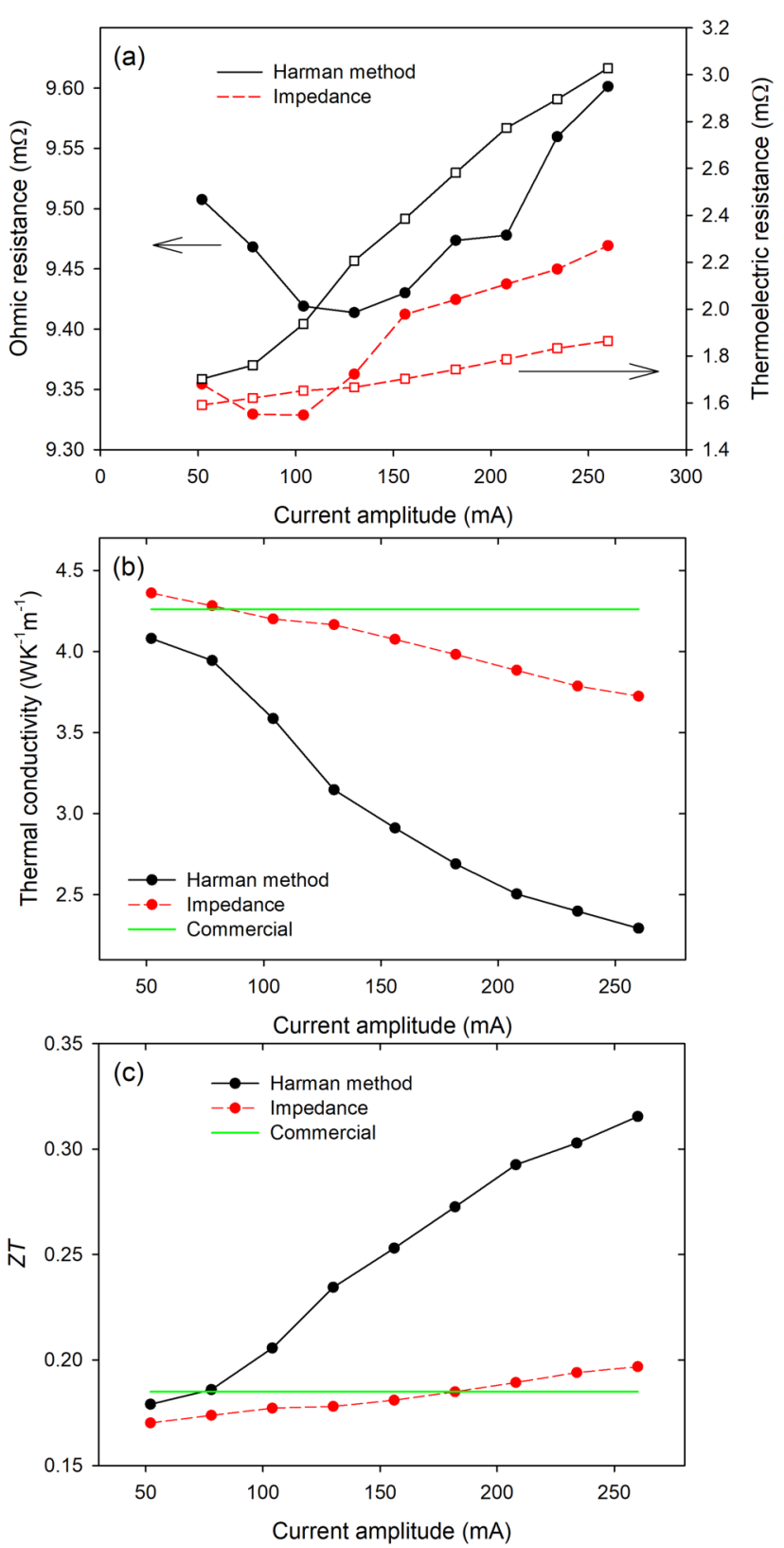

FIG. 4. Variation of (a) the ohmic, the TE resistance, (b) the ZT, and (c) the thermal conductivity with the current amplitude for the Harman (black) and impedance spectroscopy (red) methods. Commercial equipment results (green) are plotted for comparison. Measurements were performed with the SS skutterudite.

constant current. It is observed from Fig. 4(a) that the ohmic resistance does not significantly change ( $<3 \%$ variation) with the current amplitude for both methods, but the TE resistance increases with the current applied in both techniques, this increase being more pronounced for the Harman method measurements. Figures 4(b) and 4(c) show the thermal conductivity and the ZT values, respectively, obtained from the results of Fig. 4(a) and compared with the measurements from the commercial equipment. As the current amplitude increases, the thermal conductivity decreases, deviating more from the commercial equipment value at current amplitudes $>150 \mathrm{~mA}$ and $>100 \mathrm{~mA}$ for the impedance and Harman methods, respectively. This deviation is due to the variations observed in the TE resistances [Fig. 4(a)].

Furthermore, it can also be observed that results from the Harman method are more significantly affected by the influence of the current amplitude than in the impedance method, where lower deviations from the commercial equipment results were observed at large current amplitudes. For example, at a current amplitude value of $156 \mathrm{~mA}$, deviations of $4.3 \%$ and $31.7 \%$ for the impedance and Harman methods respectively, were found. This demonstrates the importance of selecting a suitable value for this parameter in order to obtain accurate results, especially in the case of the Harman method. A similar behavior was found for ZT, as shown in Fig. 4(c), where the Harman technique deviates more significantly when large currents are employed from the results obtained using the commercial equipment. For the $156 \mathrm{~mA}$ current amplitude, the deviation is $36.8 \%$, whereas for the impedance method, the maximum deviation found for the current amplitudes analyzed was $6.5 \%$, which corresponds to the largest current amplitude (260 $\mathrm{mA})$. We would like to add here that for the impedance method, it is easier to identify the optimum current amplitude to use than in the Harman method, which is the lowest possible without noise in the spectra $(104 \mathrm{~mA})$, as we already discussed in a previous work. ${ }^{12}$

\section{Frequency selection for the ac measurement}

In the Harman method, when the ohmic resistance is determined from an ac measurement, the frequency selected could influence the results. ${ }^{15}$ In order to analyze this, the magnitude $(|\mathrm{Z}|)$ and the phase $(\theta)$ of the impedance as a function of frequency is shown in Fig. 5(a) from an impedance measurement at $104 \mathrm{~mA}$ of current amplitude using the LS skutterudite. Purely ohmic behavior is considered when $\theta=0$ (around $80 \mathrm{~Hz}$ in this case), so at this condition the magnitude of the impedance provides the ohmic resistance $[\mathrm{Z}(\theta=0)=$ $R_{A C}$ ] directly. At frequencies higher or lower, capacitive or inductive effects take place and the magnitude of the impedance changes, as shown in Fig. 5(a), which may cause errors in the Harman method which cannot clearly identify the point of zero phase. Figure 5(b) shows the ZT that will result by the Harman method if a frequency different from that at zero phase is adopted, which is compared with the value from the impedance method and the commercial equipment. Although frequencies lower than the optimal produce slightly lower ZT values, no large deviations $(<0.3 \%)$ from the zero-phase point were found for all of the frequency range tested. However, this result should be treated with caution, since TE samples 

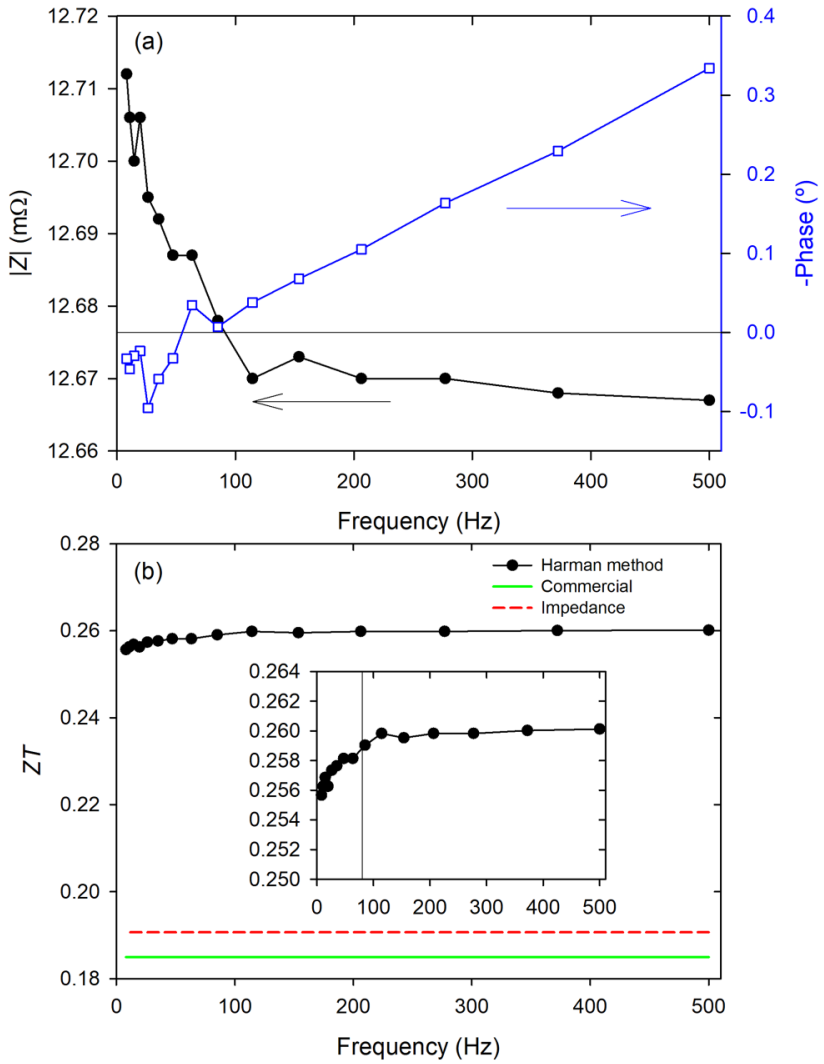

FIG. 5. (a) Impedance magnitude and phase at different frequencies in an impedance spectroscopy measurement of the LS skutterudite. (b) Variation of the $Z T$ obtained by the Harman method (black points) when different frequencies are used to obtain $R_{A C}$. $Z T$ results from the impedance technique (dotted red line) and the commercial equipment (solid green line) are also included for comparison. The inset is a magnification of the Harman method results, which includes a vertical reference line that indicates the frequency of the zero phase. with lower ohmic resistance and/or better TE properties could be more significantly affected.

\section{Use of silver paint}

It is very common to use silver paint or similar electrically conductive adhesives to attach the electric contacts to the sample under the Harman method. In addition, silver paint has also been used for impedance measurements of bismuth telluride alloys in the two-probe mode. ${ }^{11,16}$ Although silver paint has a high Ag content, it contains other chemicals that are not electrically conductive (mainly organic compounds) and requires time to cure. This could introduce additional errors into the measurements, since an electrical and thermal contact resistance ${ }^{17}$ is created at the junctions where the Peltier effect is produced. It might also lead to Joule heating at the junctions as a result of this contact resistance, potentially perturbing the heat fluxes in the system. In order to evaluate these issues related to the use of Ag paint, we have performed a series of impedance measurements using the setup of Fig. 2(c) but replacing the liquid metal by silver paint (Ref. 186-3593, RS). Figure 6 shows the impedance spectra obtained at different times after completing the curing process, which was performed following the supplier instructions $\left(5 \mathrm{~min}\right.$ at $140^{\circ} \mathrm{C}$ in an oven) once the $\mathrm{Ag}$ paint was spread at the contacts of the LS skutterudite. It can be observed that the impedance spectra jumped randomly over time with values of $R_{A C}$ much higher ( $\left.>530 \%\right)$ than in the case where liquid metal was employed. These random variations could be due to the further curing of the Ag paint, which modifies the properties of the junctions with time. Furthermore, the impedance spectra were significantly distorted, far from the suitable typical semicircle-like response. These results clearly show that using commercial silver paint could lead to inaccurate results and a material for the contact layer of higher purity and with a lack of organic compounds such as the $\mathrm{Ga}_{62} \mathrm{In}_{22} \mathrm{Sn}_{16}$ liquid metal we have employed is necessary.
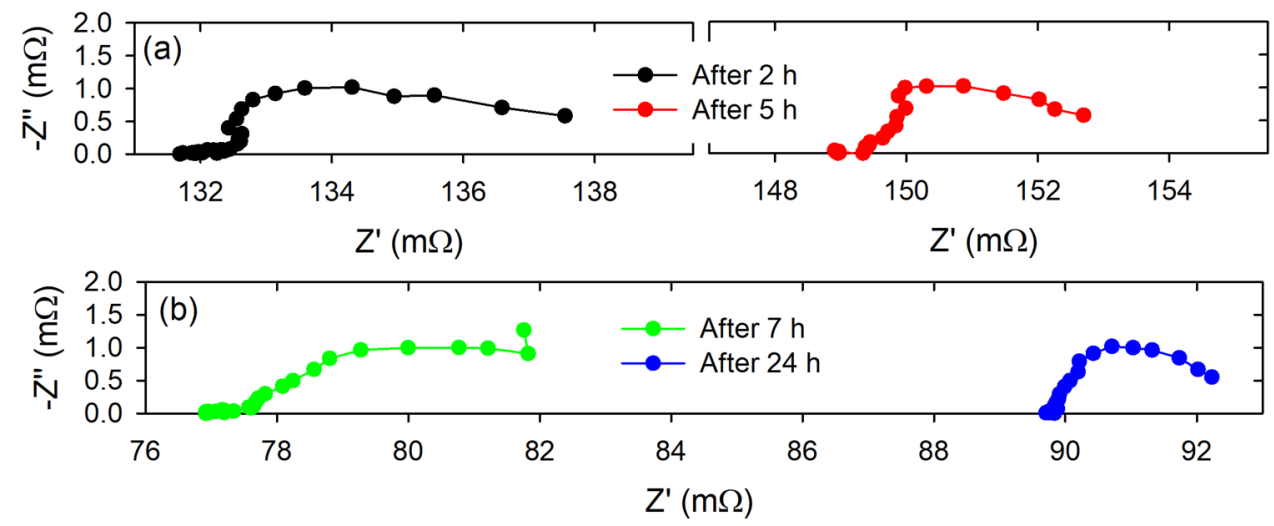

FIG. 6. Impedance spectra measured at $2 \mathrm{~h}$ (black), $5 \mathrm{~h}$ (red), $7 \mathrm{~h}$ (green), and $24 \mathrm{~h}$ (blue) after the curing process of the silver paint, which was used at the contacts between the Cu blocks and the LS skutterudite. 


\section{CONCLUSIONS}

The effect of different experimental conditions in the accuracy of the Harman and impedance spectroscopy methods to characterize TE materials has been evaluated. This was performed using a sample with TE properties previously measured with the commercial equipment. Our analysis has led to the identification of different issues that have not been clearly identified before and have shown to lead to inaccurate results. Firstly, it is important to ensure a homogeneous Peltier effect at the junctions in the setup, which is achieved using $\mathrm{Cu}$ blocks of similar cross-sectional area to that of the sample. The use of direct contact with wires produced $29.2 \%$ and $12.1 \%$ deviations with respect to the values from the commercial equipment for the thermal conductivity and ZT, respectively. Secondly, high current amplitudes can produce very large deviations in the TE properties, with a more marked effect being seen in the Harman method, where this parameter needs to be carefully selected. On the other hand, the accuracy of the measurement of the ohmic resistance in the original Harman method did not show a significant dependence on the ac frequency selected, although TE samples with lower ohmic resistance or better TE properties than the skutterudite tested could be affected more. Finally, measurements with silver paint used as a contact layer showed very high ohmic resistances with variations over time and produced distorted impedance spectra. Consequently, the use of silver paint must be avoided and an alternative material of higher purity, such as a liquid metal or solder should be selected. Only if these identified problems and sources of errors are carefully considered in the experimental setups, will accurate results be obtained by both methods.

\section{ACKNOWLEDGMENTS}

B.B.P. and J.G.C. acknowledge the financial support from the Spanish Agencia Estatal de Investigación under the Ramón y Cajal program (No. RYC-2013-13970), from the Universitat Jaume I under the Project No. UJI-A2016-08, and the technical support of Raquel Oliver Valls and José Ortega Herreros. A.V.P. and J.P.G. wish to thank the UK Engineering and Physical Sciences Research Council (EP/K019767/1) for the financial support.

\section{REFERENCES}

${ }^{\mathbf{1}}$ T. C. Harman, J. Appl. Phys. 29, 1373 (1958).

${ }^{\mathbf{2}}$ H. Iwasaki, M. Koyano, and H. Hori, Jpn. J. Appl. Phys. 41, 6606 (2002).

${ }^{3}$ H. Iwasaki, S. Y. Yokoyama, T. Tsukui, M. Koyano, H. Hori, and S. Sano, Jpn. J. Appl. Phys. 42, 3707 (2003).

${ }^{4}$ W. Kobayashi, W. Tamura, and I. Terasaki, J. Electron. Mater. 38, 964 (2009).

${ }^{5}$ B. Kwon, S. H. Baek, S. Keun Kim, and J. S. Kim, Rev. Sci. Instrum. 85, 045108 (2014).

${ }^{6}$ I. J. Roh, Y. G. Lee, M. S. Kang, J. U. Lee, S. H. Baek, S. K. Kim, B. K. Ju, D. Bin Hyun, J. S. Kim, and B. Kwon, Sci. Rep. 6, 39131 (2016).

${ }^{7}$ M. S. Kang, I. J. Roh, Y. G. Lee, S. H. Baek, S. K. Kim, B. K. Ju, D. Bin Hyun, J. S. Kim, and B. Kwon, Sci. Rep. 6, 26507 (2016).

${ }^{8}$ A. D. Downey, T. P. Hogan, and B. Cook, Rev. Sci. Instrum. 78, 93904 (2007)

${ }^{9}$ A. De Marchi and V. Giaretto, Rev. Sci. Instrum. 82, 104904 (2011).

${ }^{10}$ A. De Marchi, V. Giaretto, S. Caron, and A. Tona, J. Electron. Mater. 42, 2067 (2013).

${ }^{11}$ J. García-Cañadas and G. Min, J. Appl. Phys. 116, 174510 (2014).

${ }^{12}$ B. Beltrán-Pitarch, J. Prado-Gonjal, A. V. Powell, P. Ziolkowski, and J. García-Cañadas, J. Appl. Phys. 124, 025105 (2018).

${ }^{13}$ R. Mesalam, H. R. Williams, R. M. Ambrosi, J. García-Cañadas, and K. Stephenson, Appl. Energy 226, 1208 (2018).

${ }^{14}$ B. Beltrán-Pitarch and J. García-Cañadas, J. Appl. Phys. 123, 084505 (2018).

${ }^{15}$ Y. Hasegawa and M. Otsuka, AIP Adv. 8, 075222 (2018).

16. García-Cañadas and G. Min, AIP Adv. 6, 035008 (2016).

${ }^{17}$ D. Vasilevskiy, J. M. Simard, R. A. Masut, and S. Turenne, J. Electron. Mater. 44, 1733 (2015). 